Didáctica. Lengua y literatura

ISSN: 1130-0531

http://dx.doi.org/10.5209/DIDA.57137

\title{
Notas sobre el uso de textos de literatura filipina en español en las clases de E/LE en Filipinas
}

\author{
Jean Auguste Dominique Monsod ${ }^{1}$
}

Recibido: 6 de julio de 2016 / Aceptado: 20 de junio de 2017

Resumen. La lengua y la cultura están interrelacionadas. En Filipinas, el español constituye el núcleo de la identidad cultural poscolonial filipina. Esto se ve representado en la literatura filipina escrita en español. Sin embargo, las cambiantes relaciones de poder ya han revaluado el español en el país: aunque era una de las lenguas oficiales, ahora se enseña como lengua extranjera. Además, la literatura filipina escrita en español se ha quedado desconocida y poco leída. Este trabajo es un acercamiento teórico a la posibilidad de integrar textos de literatura filipina en español en las clases de E/LE en Filipinas. Proponemos actividades didácticas que luego vamos a estrenar en la Universidad de Filipinas Diliman. Para racionalizar tal posibilidad, partimos de dos premisas: que la universidad ofrece un programa de grado en estudios hispánicos y que siendo el español un patrimonio tanto cultural como lingüístico en el país, los estudiantes filipinos pueden aprovechar la oportunidad de aprender el español con un componente cultural más personal y experiencial. Este trabajo pretende contribuir al desarrollo de actividades y materiales que promueven el aprendizaje de español en Filipinas no como una lengua extranjera sino como una clave importante para conocer la identidad filipina en el mundo moderno.

Palabras clave: hispanismos; literatura filipina en español; E/LE; español con fines académicos; literatura en el aula

\section{[en] Notes on the use of Philippine Literature in Spanish in Teaching Spanish in the Philippines}

\begin{abstract}
Language and culture are interrelated. In the Philippines, Spanish is at the core of the postcolonial Filipino cultural identity. This found expression in Fil-hispanic literature. Yet changing networks of power relations have gradually revalued it: while it was once one of the country's official languages, it is now taught as a foreign language, its literature unread and unknown. This paper is a theoretical approach to the possibility of integrating Fil-hispanic literary texts in teaching Spanish in the Philippines. It proposes didactic activities to be piloted at the University of the Philippines Diliman. In rationalizing this possibility, these premises must be considered: that there is an undergraduate program in Hispanic Studies in this university and that with Spanish as both cultural and linguistic patrimony in the country, students can take advantage of the cultural component of the language in a way that is experiential and personally relevant. This paper aims to contribute to the development of curricula and teaching materials that promote the study of Spanish in the country not as a foreign language but an integral key to understanding the Filipino identity in the modern world.
\end{abstract}

Key words: hispanismos; Philippine Literature in Spanish; teaching Spanish as a foreign language; Spanish for academic purposes; literature in the language classroom

Departamento de Lenguas Europeas

Universidad de Filipinas Diliman

monsodjad@gmail.com 
[fr] Des notes sur l'usage de la littérature philippine en espagnol sur l'apprentissage de la langue espagnole en tant que langue étrangère aux Philippines

Résumé. La langue et la culture sont liées. Aux Philippines, l'espagnol se situe au cœur de l'identité culturelle philippine postcoloniale. Cela s'exprimait dans la littérature Fil-hispanique. Cependant, les changements des réseaux des relations de pouvoir l'ont graduellement réévaluée. Alors qu'elle était une fois une des langues officielles du pays, l'espagnol est actuellement appris en tant qu'une langue étrangère. En effet, sa littérature est devenue inconnue et non lue, reléguée à une position obscure. Cet article suit une approche théorique sur la possibilité de l'intégration des textes littéraires fil-hispanique dans l'apprentissage de la langue espagnole aux Philippines. Il propose des activités didactiques à être effectuées à l'Université des Philippines Diliman. Afin de rationaliser cette possibilité, il faut considérer les prémisses suivantes: qu'il existe un programme de baccalauréat en littérature hispanophone dans l'université et que l'Espagnol en tant que le patrimoine culturel et linguistique du pays, les étudiants peuvent profiter sur l'élément culturel de la langue d'une façon expérientielle et significative. Cet article a pour but de contribuer au développement du programme d'étude et des matériels pédagogiques qui promeuvent l'apprentissage de l'Espagnol au pays non pas comme une langue étrangère mais au contraire, en tant qu'un élément intégrant pour comprendre l'identité philippine à l'époque moderne.

Mots-clés : la littérature philippine en espagnol; l'apprentissage de l'espagnol en tant que langue étrangère; l'espagnol à des fins académiques; la littérature au sein d'une salle des langues

Sumario: 1. Introducción. 2. El uso de la literatura en las clases de E/LE. 3. La literatura filipina en español. 4. La Universidad de Filipinas y el programa de grado BA European Languages. 5. Las competencias. 5.1. Las competencias lingüísticas. 5.2. La competencia literaria. 5.3. La competencia intercultural. 6. Enfoque metodológico y tipología de actividades. 6.1. La enseñanza de español con fines académicos. 6.2. El aprendizaje basado en contenidos. 6.3. El español como patrimonio lingüístico en Filipinas. 6.4. Tipología de actividades. 6.5. La secuencia de las actividades. 7. Conclusiones. 8. Bibliografía.

Cómo citar: Dominique Monsond, J.A. (2017). Notas sobre el uso de textos de literatura filipina en español en las clases de E/LE en Filipinas Didáctica. Lengua y literatura, 29, 2017, 189-206.

\section{Introducción}

A la hora de hablar del potencial didáctico de los textos literarios en el aula de lenguas extranjeras, muchos autores coinciden en que es muy limitado si se tienen en cuenta la complejidad estructural y el uso muy particular del lenguaje literario. Sin embargo, la literatura conlleva múltiples posibilidades dentro del aula de lenguas extranjeras. En realidad, la dificultad de los textos literarios en el aula está modulada por las actividades didácticas que se elaboren en torno a dichos textos, que se rigen tanto por las necesidades de los alumnos como por el nivel de aprendizaje de los mismos (Juárez, 1996, 277). Además, la complejidad del lenguaje literario no importa si los profesores somos capaces de adaptar las actividades a los alumnos.

Nuestro objetivo es proponer un fondo teórico para el diseño de actividades que hagan uso de fragmentos de textos de literatura filipina en español para enseñar español a los filipinos. Vamos a estrenar estas actividades en la Universidad de Filipinas Diliman donde se ofrece un programa de grado en estudios hispánicos (Bachelor of 
Arts in European Languages - Spanish). El propósito de integrar los textos literarios en nuestras clases se debe a que, en primer lugar, los textos literarios son lecturas obligatorias en las clases de cultura de nuestro programa y es conveniente entrenar a los alumnos a la lectura de estos textos para prepararlos a la lectura de obras más largas y obras enteras en posteriores cursos. En segundo lugar, los textos literarios contribuyen en el desarrollo de hábitos de escribir bien, los cuales han de tener cuando empiezan a cursar las clases de cultura.

Mientras tanto, las finalidades para usar textos de literatura filipina en español en nuestras clases se deben a la realidad cultural de los alumnos filipinos. En 2012, el gobierno del país introdujo el sistema educativo K-12, un importante salto en la educación básica, que incluyó el nuevo Special Program in Foreign Language (SPFL). Antes de este gran cambio, solamente las universidades ofrecían clases de lenguas extranjeras como asignaturas optativas, pero ahora las escuelas secundarias poco a poco van incorporando las lenguas extranjeras a sus currículos. Según SEAMEO-INNOTECH (2014, s.p.), una organización internacional cuyo objetivo principal es promocionar la investigación en el campo de nuevos métodos pedagógicos, el español es la lengua con más estudiantes bajo el SPFL. Este hecho lo consideran como "una inversión prudente que tiene como fin restaurar los lazos existentes entre España y Filipinas desde hace cuatro siglos". El mismo sentimiento motivado por la vuelta de español en las escuelas filipinas fue expresado por otras organizaciones e interesados en la educación. Han usado palabras como renacimiento, resurgimiento y recuperación al describir el aprendizaje de español como una lengua que forma una gran parte de nuestro pasado nacional y como un elemento esencial de la identidad filipina. No obstante, hemos de tener en cuenta que muy pocas personas siguen hablando español en Filipinas y los documentos de los hechos históricos de la época colonial son inaccesibles en nuestro tiempo. Frente a esa realidad, podemos recurrir a los textos de literatura filipina en español para ayudar a los alumnos a conocer el papel que desempeñó la cultura española en la historia y desarrollo del país. A la larga, la apreciación por la literatura filipina en español tiene el potencial de impulsar a los alumnos a seguir una carrera en la traducción y enseñanza de esta literatura en peligro de extinción.

\section{El uso de la literatura en las clases de E/LE}

Para Collie y Slater $(1987,4)$, los textos literarios tienen las siguientes funciones en el aprendizaje de una lengua extranjera. Por un lado, proporcionan una vasta cantidad de información sobre la cultura e historia de los hablantes. Por otro lado, contienen muestras y rasgos de la lengua, por ejemplo, el léxico y las estructuras gramaticales integrados dentro de un contexto de uso.

El texto literario puede ser considerado como fuente de información cultural para los aprendientes de una lengua extranjera. Como afirman Afonso y Cámara $(2009,301)$ :

Los textos literarios a veces pueden servirnos para adentrarnos en determinados ámbitos de la vida social de un país, para acercarnos a una época particular de la historia de ese país, como reflejo del pensamiento y sentimiento de una determinada clase social, o como documento histórico. 
Entre las diferentes funciones que desempeñan los textos literarios en el aula de lenguas extranjeras, se puede distinguir su papel como muestra de la lengua. Al respecto, según Collie y Slater $(1987,4)$, "al leer textos literarios, los estudiantes tienen que hacer frente al lenguaje pensado para hablantes nativos y, de este modo, adquieren una mayor familiaridad con una gran variedad de usos lingüísticos". Asimismo, Afonso y Cámara $(2009,303)$ dicen que los textos literarios se pueden utilizar "para complementar el aprendizaje de determinados aspectos lingüísticos, tales como el léxico, la sintaxis, los marcadores del discurso, la fonética, etc.". Esta riqueza lingüística que aportan los textos literarios resulta ser muy útil si se tiene en cuenta que "en muchos casos el único input que reciben nuestros alumnos viene del manual y de nosotros mismos" (Moyano, 2008, 83). Además, si se considera que en los países en los que los alumnos no tienen muchas oportunidades para usar la lengua fuera del aula e interactuar con hablantes nativos, probablemente nos enfrentaremos al problema de cómo ampliar el vocabulario. En estas situaciones, consideramos que podemos recurrir a los textos literarios para introducir nuevas palabras, así como para presentar "estructuras sintácticas, variaciones estilísticas y formas de conectar ideas que no encontramos en la lengua oral" (Moyano, 2008, 83).

Por otro lado, al referimos al carácter motivador de los textos literarios en el aula, debemos hacer alusión a Acquaroni $(2007,59)$ para quien el uso de la literatura en la enseñanza de español facilita "la adquisición de hábitos de lectura, la capacidad de disfrutar y de comprender diversos textos literarios, el conocimiento de algunas de las obras y de los autores más representativos y la capacidad para su apreciación estética".

\section{La literatura filipina en español}

Según Donoso y Gallo $(2011,14)$, por literatura filipina en español se entiende "la literatura producida por filipinos en español, esto es, siendo Filipinas ya una entidad nacional o en su defecto una nacionalidad aspirada". En términos cronológicos, se refiere a la producción literaria decimonónica y novecentista en el país.

El crepúsculo de la ocupación española en Filipinas a finales del siglo XIX vio una prolífica producción literaria tanto crítica como revolucionaria. Esto es debido a que los escritores que produjeron obras en español eran las figuras principales del movimiento propagandista, expatriados que vivían en España donde pedían reformas como la representación política de Filipinas en el Parlamento español, la secularización y la garantía de la libertad de expresión y asociación. Después, la llegada de los norteamericanos significó el cambio en la potencia colonial dominante en el país y dio lugar, si bien es cierto que irónicamente, al florecimiento literario que más tarde llegaría a conocerse como La edad de oro de la literatura filipina escrita en castellano (1903-1942). Los temas de la mayoría de las obras durante ese periodo eran la patria, la valoración de lo nativo y un profundo anhelo por la madre España. ${ }^{2}$

Para un acercamiento al estudio de la literatura filipina en español, recomendamos los siguientes trabajos: (1) Mariñas, Luis. La literatura filipina en castellano. Madrid: Editora Nacional, 1974. (2) Alinea, Estanislao. "Philippine Literature in Spanish from the Literature of Protest to Efflorescence." En Brown, Heritage: Essays on Philippine Cultural Tradition and Literature, editado por Antonio G. Manuud. Quezon City: Ateneo de Manila University Press, 1967. (3) Ortuño, Rocío. Literatura filipina en español. Biblioteca Virtual Miguel de 
Hoy en día, puesto que la gran mayoría de la población filipina ya no habla español, la traducción es imprescindible si se espera que los filipinos se acerquen a la literatura filipina en español. Debemos prestar atención, consecuentemente, a la necesidad de salvarla del peligro de extinción, considerando que, según el Instituto Cervantes de Manila (2015, s.p.), "esta literatura por descubrir, abocada a la tragedia de devenir un corpus sin lectores, es, a pesar de su interés, la gran desconocida tanto en Filipinas como en los países hispanohablantes".

\section{La universidad de Filipinas y el programa de grado Ba European Languages}

Una mirada retrospectiva a la historia de la enseñanza de español en Filipinas nos dirá que a lo largo de los años la lengua como asignatura ha sufrido muchos cambios y se ha alternado entre ser obligatoria y electiva. El profesor Wystan de la Peña de la Universidad de Filipinas $(2011,61)$, hablando sobre el prestigio y valor económico de los que goza la lengua española en el país, dice que la lengua actualmente está experimentando un boom en su enseñanza y aprendizaje. Sin embargo, debemos reconocer que la enseñanza de español en Filipinas está en un proceso de continuo desarrollo cambiante según las necesidades y demandas de la gente del país.

El español fue una asignatura obligatoria durante los años cincuenta, una asignatura ( 24 créditos en total) que tenían que tomar los estudiantes universitarios durante cuatro o cinco años. Sin embargo, debido a la situación política del país de los años 60 y 70 - fruto del renacimiento del nacionalismo filipino-, se creó la idea negativa de que el español era lengua de los colonizadores (De la Peña, 2011, 61). Esta idea puso en marcha una sucesión de acontecimientos: el español perdió su oficialidad con la Constitución de 1987 del gobierno de la presidenta Corazón C. Aquino y, consecuentemente, el Departamento de Educación del país eliminó la obligatoriedad de las asignaturas de español. Por si fuera poco, las únicas tres universidades que siguieron ofertando español le restaron importancia al ponerla como optativa y con solo 12 créditos.

Hoy las clases de español en Filipinas se ven propulsadas por dos motivos: la formación de profesores de español de secundaria y de universidades y la enseñanza de español con fines laborales.

Por un lado, existe la necesidad real de que los profesores de español en el país estén bien formados debido a que, como afirma De la Peña $(2011,63)$, el fracaso de la enseñanza de español en el periodo previo a la Constitución de 1987 fue causado principalmente por la manera en la que se enseñaba en las escuelas. Los profesores contratados "hablaban español, sí; pero no lo enseñaban como una lengua viva, ni como una lengua de comunicación en el mundo".

No obstante, en estos momentos asistimos a un gran cambio, gracias al esfuerzo y colaboración del Instituto Cervantes de Manila, la Agencia Española de Cooperación Internacional para el Desarrollo (AECID) y la Asesoría de Educación de la Embajada de España en Filipinas, quienes han organizado una formación lingüística intensiva que tiene lugar durante las vacaciones veraniegas en el país para formar futuros profesores de secundaria.

Cervantes. 2014. Disponible en: http://www.cervantesvirtual.com/portales/literatura_filipina_en_espanol/literatura_filipina_espanol/ 
La Universidad de Filipinas también ha asumido la tarea de formar profesores de español dentro del programa de grado B.A. EuropeanLanguages. Es un programa que pretende dotar a sus alumnos de los conocimientos necesarios para poder enseñar una lengua extranjera y su literatura, en nuestro caso, el español (Bautista, 2008, $21)$. El profesor De la Peña $(2008,14)$ considera que una de las fuerzas que impulsan la labor de la Universidad de Filipinas en la enseñanza-aprendizaje del español es el discurso intelectual relacionado con el estudio del patrimonio hispánico en Filipinas. Algunos de los proyectos son traducciones de documentos, tanto literarios como históricos, ponencias sobre la literatura filipina escrita en español en conferencias nacionales e internacionales, así como montajes y reproducciones de adaptaciones teatrales y cinematográficas de obras literarias. No puede negarse, por lo tanto, que una gran parte de la labor relacionada con el español en la universidad gira en torno a la traducción y la literatura.

B.A. European Languages (especialidad en español) es un programa de cuatro años. Se divide en dos fases principales: la fase de lengua y la fase de cultura. A los alumnos se les presentan los contenidos lingüísticos en las seis primeras asignaturas: Español 10, 11, 12, 13, 14 y 15. Estas clases corresponden a un cierto nivel de dominio lingüístico: Español 10 (A1), Español 11 (A2), Español 12-13 (B1) y Español 14-15 (B2).

Una vez que han completado seis asignaturas de lengua y han alcanzado el nivel B2, ya pueden pasar a las tres clases de refuerzo de lengua. Es en estas clases de refuerzo donde pretendemos utilizar las actividades que vamos a diseñar: Español 30-31 y Español 40. Las asignaturas Español 30-31 están diseñadas para desarrollar la expresión oral, y las lecciones están organizadas por funciones lingüísticas como argumentar, dar opinión, estructurar un discurso, etc. Los contenidos lingüísticos que corresponden a estas funciones están tomados del programa de las asignaturas anteriores, Español 14 y Español 15. Por otro lado, la asignatura Español 40 consiste en la revisión y consolidación de los contenidos gramaticales aprendidos en las clases de lengua anteriores. A los alumnos se les presentan las estructuras gramaticales, junto con sus aplicaciones prácticas: por medio de comunicación oral o informes escritos.

Una vez que han completado las nueve asignaturas de lengua, se considera que los alumnos ya son capaces de leer textos lingüísticamente complejos escritos en español y que, por lo tanto, ya pueden pasar a la segunda fase del programa. En estas clases, los estudiantes trabajan con lecturas asignadas como textos literarios, ensayos y artículos académicos. También elaboran informes y presentaciones orales, así como escritos académicos sobre diversos temas relacionados con la cultura.

En el último año del programa, los estudiantes, bajo la tutela de un asesor, elaboran un proyecto fin de carrera vinculado a un tema que han estudiado en las clases de cultura. La mayoría de los trabajos que realizan los alumnos consiste en el análisis de errores gramaticales, análisis de obras literarias y estudios sobre algunos aspectos de la cultura como la gastronomía, el cine y las telenovelas. 


\section{Las competencias}

Como acabamos de explicar, el programa B.A. European Languages-Spanish tiene una naturaleza polifacética debido a que involucra muchos conocimientos, habilidades y destrezas: las competencias lingüísticas, las habilidades de lectura y escritura, la creación e interpretación de textos y la adquisición de conocimientos sobre varias áreas como historia, geografía, lingüística, literatura y traducción. Por consiguiente, las competencias que esperamos desarrollar con nuestras actividades se rigen por estas exigencias del programa.

\subsection{Las competencias lingüísticas}

Consideramos que las competencias lingüísticas, es decir, la léxica y la gramatical, son indispensables para cualquier estudiante del programa, especialmente porque van a enfrentarse a varios textos tanto académicos como literarios a lo largo de sus estudios. Hemos basado nuestra afirmación en Hosenfeld (1979), quien dice que dos factores posibilitan la comprensión de un texto: el lector (¿Le interesa el texto? ¿Por qué lo lee? ¿Conoce bien el tema del texto?) y el texto en sí (el tipo textual, la estructura del texto, la sintaxis y el léxico). Citamos también a Grabe y Stoller $(2002,186)$, quienes parten del segundo factor y señalan que los estudiantes de una segunda lengua tienen que reconocer el $95 \%$ de las palabras en un texto para poder leerlo cómodamente.

Mientras tanto, Sitman y Lerner (1994, s.p.) resumen la importancia de usar textos literarios para facilitar el aprendizaje del vocabulario y la gramática con estas palabras:

El texto sirve de estímulo y vehículo para enseñar y practicar las estructuras y el léxico del idioma en un contexto auténtico y variado, y para aprender cómo este lenguaje -en nuestro caso el español- es hábilmente empleado, manipulado, maniobrado, por distintos autores para comunicar mensajes y explorar y expresar realidades diferentes.

\subsection{La competencia literaria}

Para Brumfit y Carter $(1986,18)$, la competencia literaria es "una interesante combinación de la conciencia lingüística, socio-cultural, histórica y semiótica". De ahí, tal y como dice Sanz $(2005,137)$, abarca muchos conocimientos y destrezas como lo lingüístico, lo histórico, los conceptos relacionados con la teoría literaria y el lenguaje literario y la habilidad de interpretación y creación. Tal naturaleza interdisciplinaria de esta competencia aparece resaltada también en Martínez Ezquerro (2014, 457-458) para quien la adquisición de la competencia literaria es un proceso complejo que involucra lo siguiente:

- la comprensión básica del texto,

- el texto mismo, que da al lector pautas para su interpretación,

- el lector, quien interpreta y valora el texto y

- el intertexto del lector, compuesto por el conjunto de saberes y estrategias lingüístico-culturales que contribuyen en la interpretación del significado. 
Dicho lo cual, la competencia literaria da lugar al "enfoque comunicativo de la literatura", que aprovecha el potencial semántico y semiótico de los textos literarios para el aprendizaje de una lengua $\mathrm{y}$, a la vez, la adquisición de conocimientos sobre varios temas de interés (González, 1992, 60).

\subsection{La competencia intercultural}

Como hemos resaltado anteriormente, el español es un patrimonio lingüístico en Filipinas. Por consiguiente, el caso de las aulas de E/LE en Filipinas no es el mismo que el de las demás aulas de E/LE en los países asiáticos. Según Ofilada $(2011,256)$ :

Los filipinos, desde una perspectiva lingüística y cultural, tienen una ventaja cognitiva en el aprendizaje del español que los demás asiáticos no poseen. Para los asiáticos, el español es totalmente una lengua extranjera (perspectiva de la alteridad). Para los filipinos, es más bien una lengua propia mas olvidada, superada, arrinconada, perdida que es preciso recuperar.

En el aula, esto supone el uso de las dos pruebas omnipresentes de tal propiedad. En primer lugar, la lengua española dejó una fuerte influencia en las lenguas autóctonas manifestada en los préstamos lingüísticos o hispanismos (Alcántara, 1998). Ellos se pueden clasificar en tres:

- las palabras que no han sufrido ningún cambio de ortografía o pronunciación (mesa, papel, blusa),

- las palabras que han cambiado de ortografía o pronunciación (zapatos $\rightarrow$ sapatos, vaso $\rightarrow$ baso, silla $\rightarrow$ silya) y

- las palabras que han cambiado de significado (siempre ["always"] $\rightarrow$ siempre ["of course"]).

En segundo lugar, como hemos detallado en el apartado 3, los escritores filipinos dejaron una considerable producción literaria escrita en español. En definitiva, creemos importante subrayar que nuestros alumnos filipinos tienen una ventaja cognitiva en las clases de E/LE. Es decir, pueden emplear la estrategia descrita por Hansejordet $(2006,4)$, la de "partir de lo que ya sepas o te imagines acerca del tema, y de las palabras que ya conozcas o adivines a partir del contexto o basándote en tu lengua materna u otras lenguas".

\section{Enfoque metodológico y tipología de actividades}

Basándonos en lo dicho hasta ahora, vamos a tener en cuenta las siguientes premisas a la hora de confeccionar nuestras actividades.

\subsection{La enseñanza de español con fines académicos}

González Alfaya (2009), en un estudio de caso que realizó sobre los perfiles y las experiencias de los estudiantes extranjeros en la Universidad de Vigo, señaló la dificul- 
tad a la que se enfrentan estos alumnos durante su estancia en la universidad, debida principalmente a sus deficiencias en el dominio de la lengua en la que se imparten sus clases. Pastor $(2006,14)$, partiendo del hecho de que hay cada vez más estudiantes extranjeros que entran en las universidades españolas, subraya la importancia de enseñar español no desde una perspectiva comunicativa general sino como lengua de instrucción para ayudar a los alumnos a aprovechar su currículo en la universidad.

Enmarcamos el programa BA European Languages dentro de la enseñanza de español con fines académicos de acuerdo con la misma autora arriba citada $(2006,5)$ quien nos proporciona esta definición:

La enseñanza de una segunda lengua con fines académicos puede entenderse como una tarea propedéutica que prepara al alumno para entender y asimilar mejor un determinado contenido de su currículum, impartido en una lengua que no es la suya materna, así como para interactuar a través de ella en el aula.

¿Cómo se caracteriza la enseñanza de una lengua con fines académicos? Para el Modern Language Association (MLA) (2007, s.p.) la enseñanza de lenguas extranjeras en el nivel superior se debe impartir teniendo en cuenta lo siguiente. En primer lugar, se utilizan la literatura, el cine y otros medios de comunicación para enseñar a los alumnos la interpretación y la traducción, la consciencia política e histórica, la sensibilidad social y la percepción estética, entre otras cosas. En segundo lugar, los alumnos deben adquirir un conocimiento básico de la historia, geografía, cultura y literatura de la gente que habla la lengua meta. En último lugar, ellos deben ser capaces de realizar investigaciones en la lengua meta.

\subsection{El aprendizaje basado en contenidos}

Como hemos recalcado anteriormente, las clases de lengua de nuestro programa constituyen la fase preparatoria hacia la tarea académica exigida por la enseñanza superior. De ahí, necesitamos entrenar a los alumnos a usar la lengua que están aprendiendo para poder desenvolverse en las posteriores clases de cultura, las cuales involucran el comentario y la lectura de textos sobre varios temas de interés. Esto se basa principalmente en el aprendizaje basado en contenidos o content-based learning, en el que el centro de cada lección se encuentraen el contenido del texto. De esta manera, los alumnos puedan usar la lengua que están aprendiendo como una herramienta para adquirir conocimientos y a la vez para desarrollar su competencia lingüística (British Council, 2003, s.p.).

En la misma línea, Lain (1975) enumera los tres aspectos fundamentales de la didáctica de la literatura:

(1) lo que el texto quiere decir gramaticalmente, es decir, el texto como muestra de lengua,

(2) lo que el texto quiere decir históricamente o lo que pueden aprender los alumnos sobre su visión del mundo descrita en el texto y

(3) lo que el texto quiere decir personalmente, es decir, las relaciones que los estudiantes pueden encontrar o crear entre sus experiencias personales y las situaciones y personajes representados en el texto. 
Vicente $(2011,104)$, por su parte, resume lo que ha dicho Lain, destacando que mediante esta manera de tratar la literatura en el aula, los alumnos pueden desarrollar sus conocimientos gramaticales a la vez que usan la lengua para hablar del contenido de los textos literarios "de manera comunicativa y experiencial".

\subsection{El español como patrimonio lingüístico en Filipinas}

El español como patrimonio lingüístico, de hecho, ya se ha llevado a las aulas filipinas mediante diferentes actividades y propuestas didácticas. Salazar (2013), ha sugerido el aprovechamiento de los hispanismos para facilitar el aprendizaje del vocabulario y el desarrollo de la comprensión lectora, así como para hacer a los alumnos conscientes de cómo ciertas palabras en español han entrado en el léxico de las diferentes lenguas autóctonas. Afonso y Cámara (2009), por su parte, han propuesto el uso de textos de literatura filipina en español pero solo como modelo de la lengua para facilitar el aprendizaje del vocabulario y la gramática. Ahora bien, ¿cómo podemos aprovechar la literatura filipina en español para enseñar ambos la lengua y el contenido? ¿Qué contenidos de los textos literarios debemos subrayar para conseguir nuestro objetivo de hacer a nuestros alumnos más conscientes de la influencia de español en nuestra vida e historia?

Según Dema y Moeller $(2012,79)$ no debemos enseñar la cultura como información suelta o "bits of trivia". Añade Kramsch $(2013,66)$ que tal manera de llevar la cultura al aula es una forma de enseñanza que carece de "profundidad histórica". Consideramos que esta profundidad histórica a la que alude la autora resultaría ser esencial en la enseñanza de español en Filipinas por estas razones. En primer lugar, queremos que nuestros alumnos sean,tal y como dicen Barnes-Karol y Broner (2010, 423-24), usuarios capaces de hacer preguntas significativas sobre su cultura propia y la cultura de la lengua meta, así como examinar el mundo con ojo crítico. En segundo lugar, según Kramsch $(2009,238)$, a la hora de leer los textos literarios en la clase, queremos que los alumnos señalen a su atención "las resonancias históricas de las palabras", es decir, el significado de las palabras, así como las costumbres y perspectivas que dan lugar a tales significados. En último lugar, si nuestros alumnos son conscientes de los lazos culturales y lingüísticos entre España y Filipinas, van a comprender qué implica usar una lengua, ya sea materna, segunda y extranjera, y como dice Bryam $(2016,7)$, van a ser conscientes de la importancia de cualquier lengua a su identidad personal y social.

\subsection{Tipología de actividades}

A continuación presentamos la tipología de las actividades según la competencia que queremos desarrollar. Estos son los tipos de actividades que desarrollan las competencias léxica y gramatical:

1. Relacionar palabras con sus definiciones.

2. Escribir el significado de las palabras según cómo se han usado en el texto.

3. Relacionar palabras con imágenes.

4. Construir frases utilizando unidades/expresiones relacionadas con una función lingüística. 
5. Construir frases siguiendo un modelo tomado del texto.

6. Explicar los usos de una estructura gramatical.

Las actividades que desarrollan la competencia intercultural son:

1. Subrayar o identificar los hispanismos en el texto y decir qué significan en español y en la lengua autóctona.

2. Hablar de la forma de vida durante los tiempos de España en Filipinas (periodo en el que transcurren los relatos).

Por último, las actividades que desarrollan la competencia literaria son:

1. Hablar y compartir conocimientos sobre los autores representativos en la literatura filipina en español.

2. Contestar preguntas de comprensión del contenido del texto.

\subsection{La secuencia de las actividades}

A partir de lo aquí expuesto, ahora vamos a describir las actividades de nuestra propuesta. Vamos a dividir la secuencia didáctica en tres partes:

1. Precalentamiento o pre-lectura

Vamos a empezar a trabajar los contenidos lingüísticos desde esta parte de la lección. Las actividades van a consistir en interacciones entre el profesor y la clase. En esta parte el profesor proporciona las unidades o expresiones que pueden utilizar los alumnos, de acuerdo con una función lingüística, por ejemplo, expresar qué se recuerda. El precalentamiento tiene como objetivos lo siguiente:

a) permitir que los alumnos activen sus conocimientos previos y hagan uso de sus propias experiencias personales. A continuación tenemos un ejemplo:

Decimos a los alumnos que vamos a leer un fragmento de Cuentos de Juana de Adelina Gurrea Monasterio. ${ }^{3}$

¿Creciste aquí en Manila o en una provincia fuera de la capital? ¿Hay recuerdos de tu juventud de los que siempre te acuerdas? ¿Tenías un programa de televisión favorito? ¿O un amigo con quien siempre estabas? Relaciona las siguientes palabras con algún recuerdo de tu juventud: juegos, canciones, asignatura, lugar, libro, amigos, comida, programa de televisión. Puedes seguir estos modelos:

Cuando era pequeño/a, tenía un favorito...

\footnotetext{
Habría que hacer mención de un artículo en el que se elabora, entre otras, una secuencia didáctica en torno también a Cuentos de Juana. En el artículo, los autores presentan sus reflexiones sobre la posibilidad de utilizar textos de literatura española e hispanofilipina en las clases de E/LE mientras que nuestro trabajo propone algo más específico, que es el uso de textos de literatura filipina en español para un público particular: los alumnos de un programa de grado en estudios hispánicos en universidades en Filipinas. Consideramos que este tipo de destinatario requiere un currículo especializado, prestando especial atención al desarrollo de conocimientos y destrezas particulares. González, Verónica y María José Lobón, "Literatura filipina e hispano-filipina en la clase de E/LE" (ponencia presentada en Competencias y Estrategias Docentes en el Contexto de Asia-Pacífico, Manila, noviembre 2011), http://bit.ly/1VYMUBu.
} 
De pequeño/a...

De joven...

¿Recuerdas? El imperfecto se utiliza para hablar de acciones habituales, acciones realizadas regularmente o costumbres en el pasado, por lo que se utiliza para hablar de cosas que hacíamos durante la juventud. Para hablar de acciones habituales en el pasado, podemos usar también soler + infinitivo. Por ejemplo: Cuando era pequeño solía ir al parque con mis hermanos.

b) inducir a los alumnos a usar la lengua meta para hablar de un cierto tema relacionado con la cultura, por ejemplo, algunos eventos históricos o tradiciones que aparezcan en el texto o cómo piensan que era la vida durante los tiempos del autor de la obra. Tenemos como ejemplo la siguiente actividad:

Vamos a hablar del héroe nacional José Rizal y su obra fundamental, Noli me tangere. [Proyectamos algunas capturas de pantalla de la adaptación al cine dirigida por Gerardo de León en 1961]. ¿Qué te sugieren? ¿Sabes en qué siglo transcurren los eventos? ¿Qué elementos ves que te informan sobre la vida durante esa época? Coméntalo a tu profesor.

En la escuela secundaria, ¿leíste toda la novela o solo fragmentos? ¿Qué recuerdas de la novela? ¿Quién es el personaje que más te llamó la atención? Coméntalo a un compañero.

Puedes utilizar estas unidades:

Recuerdo (perfectamente) haber leído que...

No me he olvidado de que...

Me suena que...

Me interesa el personaje... porque...

Me gusta el personaje... porque...

El personaje que más me ha llamado la atención es... porque...

2. Lectura de textos

Para las actividades durante la lectura de textos, insistimos en sus dos funciones. Por una parte, ayudan a los alumnos a comprender el propio contenido del texto y, por otra parte, permiten que los alumnos practiquen contenidos léxicos y gramaticales. Las actividades durante la lectura se realizan de la siguiente manera:

- Los alumnos leen el texto silenciosamente.

- Hacen actividades de comprensión global, por ejemplo, preguntas sobre las ideas principales del texto o preguntas de Verdadero o Falso.

- El profesor presenta los contenidos léxicos y gramaticales que van a trabajar y los alumnos tienen que buscar y subrayar estos elementos en el texto.

- Los alumnos identifican palabras complicadas pero parecidas al filipino o a sus lenguas nativas para saber qué significan en español.

\section{Lectura de texto}

Como cuando yo conocí a Juana ésta aún no era vieja, debemos deducir que estuvo sirviendo siempre en la familia. Tenía el remoquete de Baltimore. Nació tal 
remoquete del nombre de uno de los acorazados norteamericanos que hicieron la parodia de batirse contra la carcomida y resquebrajada escuadra española en la bahía de Cavite. Juana había sido muy flamencota y muy gruesa, y por su amplitud se la comparó con el voluminoso acorazado americano. Cuando yo me di cuenta de la personalidad de Juana, ésta ya había mermado sus carnes; era muy oscura de color, nariz marcadamente aplastada, ojos pequeños y una boca grande, sin la albura de los dientes debajo de sus labios marchitos. Nació con la dentadura blanca, pero el buyo fue depositando una pátina roja sobre el marfil, y éste tenía ya el color de sangre renegrida.

Para un hombre occidental recién llegado de Europa o de América, el aspecto de tal boca sería repugnante, pero los que allí nacimos y los que llevan muchos años de residencia en el país nos familiarizamos con el buyo y su colorido desagradable, de tal modo, que yo nunca rehuí, de niña, un beso de su boca y de su ternura. Otros, sin ser niños, tampoco los rehuían, porque Juana, fea como era, tenía un atractivo especial para los hombres malayos o europeos.

En mi hogar era una institución. Resultaba menos ignorante que el resto de las mujeres del rancho, por su trato con los españoles en casa de mi abuela y en la de mis padres. Hablaba el castellano, pero se olvidaba de él tan pronto como se la reprochaba alguna mala acción o falta en el cumplimiento de su deber. Entonces no se encontraba modo de evitar que diese sus pródigas explicaciones en el dialecto visayo. Como todo filipino, escuchaba las órdenes e instrucciones que se le daban con un gesto de complacencia, cual si fuese a cumplirlas al pie de la letra, y luego hacía lo que mejor le venía en gana. En el momento de las explicaciones, largaba su discursito en visaya; pero a veces se encontraba con réplicas que eran más que palabras. Cosas de la colonización. Para Juana no era denigrante el golpe en la mejilla o el cachete. Si era el ama quien se los daba, el ama podía hacerlo.

A nosotros parecía querernos y -bien o mal- cuidó de todos los hermanos. Por las noches, antes de acostarnos, o en las siestas cálidas y soporíferas, mientras planchaba la blancura de las ropas tropicales, nos contaba cuentos de reyes y princesas españolas o los terroríficos de duendes malayos.

- Cuentos de Juana, Adelina Gurrea Monasterio

Actividad 1

Contesta a las siguientes preguntas.

1) ¿De qué trata el fragmento? ¿Qué cualidades de Juana te han llamado la atención? ¿Por qué?

2) ¿Cómo se llamaba ese lugar donde transcurrían todos los cuentos de Juana?

3) ¿Entiendes la palabra remoquete? Según el narrador, Juana "tenía el remoquete de Baltimore". ¿Por qué le dieron ese apodo?

4) ¿Cómo se describieron los aspectos del rostro de Juana? Con un compañero, dibuja cómo la imagináis. ¿Creéis que corresponde con nuestro concepto tradicional de belleza? Justificad vuestra respuesta. 
5) ¿Entiendes la palabra buyo ${ }^{4}$ ? ¿Qué palabras utilizó el narrador para describir qué hace el buyo al color de los dientes de Juana?

6) Según el narrador, Juana "era una institución”. ¿Qué significa "ser una institución”? ¿Por qué se describe Juana de esta manera?

7) ¿Qué lenguas hablaba Juana?

8) ¿De qué se trataban los cuentos que Juana solía contar?

9) La narradora describe el carácter de Juana de una manera única: no utiliza meros adjetivos sino que habla de las cosas que solía hacer y cómo se relacionaba con la gente. De ahí, podemos deducir cómo era su carácter. Por ejemplo, ¿qué nos dice esta descripción sobre el carácter de Juana?: “...escuchaba las órdenes e instrucciones que se le daban con un gesto de complacencia... luego hacía lo que mejor le venía en gana”.

Actividad 2

Escribir una descripción de manera creativa no solo consiste en decir el mensaje al lector sino además en describir situaciones para transmitir un cierto mensaje o evocar una emoción. Observa estas dos frases:

1a) Carla y su padre estaban muy apegados.

1b) Carla era la regalona de su padre. En la universidad, viviendo miles de kilómetros fuera de su pueblo natal, Carla contemplaba antes de dormir la foto de su padre colgada en la pared de su dormitorio.

La primera frase transmite el mensaje directamente mientras que la segunda frase nos hace deducir que Carla y su padre estaban muy apegados. La segunda nos ofrece una imagen con la que podemos deducir el mensaje que se quiere transmitir. Ahora, ¿cómo describirías una cosa, un lugar o una persona que posee estas características?

Como en las frases de la actividad anterior, utiliza el imperfecto.

1) repugnante

2) desagradable

3) pródigo

4) miedoso

5) optimista

6) impío

7) travieso

8) fuerte

9) sucio

10) inteligente

\section{Tarea final}

Esta consiste en un trabajo escrito en el que se reúnen de manera creativa los contenidos que han aprendido los alumnos durante la secuencia. Se realiza de forma individual o en grupos de 3 o 4 . A continuación tenemos un ejemplo:

$4 \quad$ El profesor puede preguntar si algunos de los alumnos han ido una vez a Mountain Province, en el norte de Filipinas, donde la gente masca el buyo, lo cual da una coloración roja a la saliva. 
¿Qué tiempo verbal predomina en el texto de Adelina? ¿Por qué se emplea este tiempo verbal?

Como ves en el texto, la narradora ha utilizado el imperfecto para hablar de los aspectos y características de una persona que conocía en su juventud. Ella nos está contando cómo era Juana según lo que recuerda. Seguro que cuando eras pequeño/a, conocías a una persona que nunca has olvidado. ¿Cómo era? ¿Qué recuerdas de él o ella? Escribe un texto describiendo a esa persona inolvidable.

\section{Conclusiones}

Hemos tratado de dejar patente a lo largo de nuestro trabajo que la literatura filipina en español puede ser muy enriquecedora en el contexto de E/LE en Filipinas, teniendo en cuenta que los alumnos filipinos pueden aprovecharla de manera personal y experiencial. Por decirlo de otro modo, el uso de la literatura filipina en español en las clases de E/LE en Filipinas permite que los alumnos aprendan sobre el patrimonio hispánico en el país - manifestado en lo histórico, lo lingüístico, lo artístico y lo cultural- que no podrían aprender de otra manera. Y es así, el aprendizaje de español llega a ser el redescubrimiento de esa parte de la historia filipina que queda inaccesible para la gente ya no hispanohablante del país.

En suma, a través de las actividades que hemos diseñado, hemos intentado dar a conocer que los profesores de español en Filipinas pueden elaborar, a partir de textos literarios, actividades contextualizadas que resulten familiares a los alumnos. Y hemos creído oportuno empezar a usar estas actividades en el programa B.A. European Languages de la Universidad de Filipinas, porque los alumnos de este programa están aprendiendo español con el propósito de poder enfrentarse a la literatura. De este modo, la utilización de textos literarios en las clases de lengua en la universidad contribuye fructuosamente a la formación lingüística y literaria.

Concluimos diciendo que aunque solo hemos podido presentar una secuencia, hay muchas más actividades que podríamos hacer. Como tareas pendientes, podemos elaborar actividades o talleres con el objetivo de desarrollar la expresión escrita, o diseñar lecciones que se utilizarán exclusivamente en las clases de conversación. Además, los alumnos pueden hacer presentaciones de las obras teatrales y convertir una novela o un cuento corto en una breve pieza teatral o un cortometraje. Estos podrían ofrecer a los alumnos un espacio para la creatividad y les permitirían adquirir una nueva o mejor comprensión de la lengua y la cultura en los textos. Podríamos contemplar también la posibilidad de crear actividades utilizando textos de literatura filipina en español en las clases de niveles más bajos. Finalmente, podríamos fomentar el uso de textos de literatura filipina en español en otras universidades filipinas, así como en las clases de lengua en las escuelas de idiomas como el Instituto Cervantes de Manila, por lo cual sería importantísimo que los profesores filipinos diseñáramos y luego publicáramos nuestros propios manuales y materiales. Lo que sabemos de cierto es que para los profesores de español en Filipinas hay muchas posibilidades de enseñar español con modalidades tanto innovadoras como única. 


\section{Bibliografía}

Acquaroni, Rosana (2007): Las palabras que no se lleva el viento: literatura y enseñanza de español como LE/L2, Madrid, Santillana Education S.L.

Afonso, Vanesa y Cámara, Rosa (2009): "El uso de la literatura hispanofilipina en el aula de ELE", ponencia presentada en El Currículo de E/LE en Asia Pacífico. I CELEAP, Manila, http://cvc.cervantes.es/ensenanza/biblioteca_ele/publicaciones_centros/pdf/manila_2009/19_aplicaciones_06.pdf. [Consulta: 23 de junio de 2016].

Alcántara, Teresita (1998): Los hispanismos en los medios de comunicación social filipinos: Estudio lingüístico, Quezon City, Sentrong Wikang Filipino, Unibersidadng Pilipinas.

Barnes-Karol, Gwendolyn. y Broner, Maggie (2010): "Using images as springboards to teach cultural perspectives in light of the ideals of the MLA Report", Foreign Language Annals, 43(3), 422-445.

Bautista, Erwin (2008): “La enseñanza de español en Filipinas", Linguae et Litterae, 6, 1823, http://journals.upd.edu.ph/index.php/lel/article/view/1772/1690. [Consulta: 20 de junio de 2016].

British Council(2003): “Content-basedlearning”, https://www.teachingenglish.org.uk/article/content-based-instruction. [Consulta 11 noviembre 2016].

Brumfit, Christopher and Carter, Ronald (1986): Literature and Language Teaching, New York, New York University Press.

Byram, Michael (2012): "Language awareness and (critical) cultural awareness - relationships, comparisons and contrasts", en Awareness Matters: Language, Culture, Literacy, Claudia Finkbeiner y AgnetaSvalberg (eds.), Routledge, 5-13.

Collie, Joanne y Slater, Stephen (1987): Literature in the language class room: A resourcebook of ideas and activities, Cambridge, Cambridge University Press.

De La Peña, Wystan (2008): "The University of the Philippines in Defense of Spanish", Linguae et Litterae, 6, 6-17. http://journals.upd.edu.ph/index.php/lel/article/view/1770/1691 [Consulta: 23 de junio de 2016].

De La Peña, Wystan (2011): "La enseñanza del español en Filipinas: repitiendo el experimento, recordando las lecciones", Perro Berde: Revista Cultural Hispano-Filipina, 2, 60-65.

Dema, Oxana y Moeller, AleidineKramer(2012): “Teaching culture in the21st centurylanguageclassroom", FacultyPublications: Department of Teaching, Learning and TeacherEducation, 181, 75-91. http://digitalcommons.unl.edu/cgi/viewcontent.cgi?article $=1176 \&$ context $=$ teachlearnfacpub [Consulta: 13 noviembre 2016].

Donoso, Isaac y Gallo, Andrea (2011): Literatura hispanofilipina actual, Madrid, Editorial Verbum.

González Alfaya, Lucila (2009): "Español como lengua extranjera con fines académicos en la Universidad de Vigo: un estudio de caso", ponencia presentada en El español en contextos especificos: enseñanza e investigación. XX Congreso Internacional de la ASE$L E$, Comillas, http://cvc.cervantes.es/ensenanza/biblioteca_ele/asele/pdf/20/20_0552.pdf [Consulta: 22 junio 2016].

González, Luís (1992): "La literatura en la educación secundaria", Signos, 7, 54-61.

Grabe, William y Stoller, Fredericka L. (2002): Teaching and Researching Reading, London, Longman.

Hansejordet, Ingunn (2006): "Trabajar con textos literarios en clase", ponencia presentada en 2006, Año del Español en Noruega. Un reto posible, Oslo, http://www.mecd.gob.es/ $\mathrm{dctm} / \mathrm{redele} /$ Material-RedEle/Numeros\%20Especiales/2006_ESP_09_Actas1 erCongre- 
so/Comunicaciones/2006_ESP_09_12Hansejordet.pdf?documentId=0901e72b80e52e76 [Consulta: 23 de junio de 2016 ].

Hosenfeld, Carol (1979): "Cindy: A Learner in Today's Foreign Language Classroom", ponencia presentada en Northeast Conference of the Teaching of Foreign Languages, Washington, DC, https://eric.ed.gov/?id=ED185837. [Consulta 9 de julio de 2017].

Instituto Cervantes de Manila (s.f.). "Clásicos hispanofilipinos", manila.cervantes.es. http:// manila.cervantes.es/es/cultura_espanol/Clasicos_hispanofilipinos/Clasicos_hispanofilipinos.htm [Consulta: 23 de junio de 2016].

Juárez, Pablo (1996): "La enseñanza de la literatura en los cursos de lengua y civilización para extranjeros", ponencia presentada en Lengua y Cultura en la Enseñanza del Español a Extranjeros. VII Congreso Internacional de la ASELE, Almagro, http://cvc.cervantes. es/ensenanza/biblioteca_ele/asele/pdf/07/07_0275.pdf [Consulta: 2 de mayo de 2016].

Kramsch, Claire (2009): "Third culture and language education" en Contemporary Applied Linguistics. Vol. 1, Vivian Cook y Li Wei (eds.), London, Continuum, 233-254.

Kramsch, Claire (2013): "Culture in Foreign Language Teaching”, Iranian Journal of Language Teaching Research, 1(1), 57-78. file://Users/dominiquemonsod/Desktop/Kramsh\%20culture $\% 20$ in $\% 20$ foreign $\% 20$ language $\% 20$ teaching\%20Kramsch.pdf [Consulta 28 junio 2016].

Laín, Pedro (1956): La aventura de leer, Madrid, Austral.

Martínez Ezquerro, Aurora (2014): "Desarrollo de destrezas comunicativas en L2: nuevos materiales didácticos", ponencia presentada en La enseñanza de ELE centrada en el alumno. XXV Congreso Internacional de la ASELE, Madrid, http://cvc.cervantes.es/ensenanza/biblioteca_ele/asele/pdf/24/24_455.pdf [Consulta: 23 de junio 2016].

Modern Language Association(2007):"Foreign Languages and Higher Education: New Structures for a Changed World", https://www.mla.org/Resources/Research/Surveys-Reports-and-Other-Documents/Teaching-Enrollments-and-Programs/ForeignLanguages-and-Higher-Education-New-Structures-for-a-Changed-World [Consulta: 22 junio 2016].

Moyano, Juan Carlos (2008): "Reflexiones sobre el uso de la literatura en la clase de ELE",Japanese Institutional Repositories Online, 125, 75-106, https://dspace.wul.waseda.ac. jp/dspace/bitstream/2065/32788/1/KyoyoShogakuKenkyu_125_Lopez.pdf [Consulta: 23 de junio de 2016].

Ofilada, Macario (2011): "Del Marco común europeo de referencia (MCER) a las aulas de ELE en Filipinas: los contenidos culturales dentro del discurso y la toma de decisiones del profesor", ponencia presentada en Competencias y Estrategias Docentes en el Contexto de Asia-Pacífico. II CELEAP, Manila, http://cvc.cervantes.es/ensenanza/biblioteca_ele/ publicaciones_centros/pdf/manila_2011/18_investigaciones_10.pdf [Consulta: 23 de junio de 2016].

Pastor, Susana (2006): "La enseñanza del español como lengua vehicular en contextos académicos", marco ELE: Revista de Didáctica Español Lengua Extranjera, 2, 1-25. http:// marcoele.com/descargas/2/pastor-contextos_academicos.pdf [Consulta: 26 junio 2017].

Salazar, Danica (s.f.): "Los hispanismos en la enseñanza del español a aprendices filipinos", Yo te diré: Revista Digital de la Asesoría de Educación en Manila, 3, 19-3. http://www. mecd.gob.es/filipinas/dms/consejerias-exteriores/filipinas/publicaciones/ytd03_2012.pdf [Consulta: 11 noviembre 2016].

Sanz, Marta (2005): "Didáctica de la literatura: el contexto en el texto y el texto en el contexto", ponencia presentada en Programa de Formación para Profesorado de ELE 20042005, Múnich, http://cvc.cervantes.es/ensenanza/biblioteca_ele/publicaciones_centros/ PDF/munich_2004-2005/02_sanz.pdf [Consulta: 9 de junio de 2016]. 
Seameo-Innotech (2014): "Spanish language program in Philippine public secondary schools", http://www.seameo-innotech.org/projects-completed/spanish-language-assessment-program/ [Consulta: 2 mayo2016].

Sitman, Rosalie y Lerner, Ivonne (1994): "Literatura hispanoamericana: herramienta de acercamiento cultural en la enseñanza del español como lengua extranjera", ponencia presentada en Tendencias Actuales en la Enseñanza del Español como Lengua Extranjera I. V Congreso Internacional de la ASELE, Santander, http://cvc.cervantes.es/ensenanza/ biblioteca_ele/asele/pdf/05/05_0225.pdf [Consulta: 5 de mayo de 2016].

Vicente, Leticia (2011): "La atención a la forma del discurso escrito de aprendices de E/LE en el contexto universitario hongkonés", ponencia presentada en IV Jornadas de Formación de Profesores de Español como Lengua Extranjera en China: Didáctica y Materiales en el Aula de E/LE, China, Pekín, http://sinoele.org/images/Congresos/IVJornadas/ Actas/vicente_100-133.pdf [Consulta: 5 de mayo de 2016]. 